\title{
Editorial
}

Nephrology

Published online: December 17, 2018

\section{Shortening the Duration of Corticosteroid Exposure in Minimal Change Disease: Can We Treat Adults Like Children?}

\author{
Andrew S. Bomback \\ Department of Medicine, Division of Nephrology, Columbia University College of Physicians and Surgeons, \\ New York, NY, USA
}

At first glance, the treatment of minimal change disease (MCD) appears straightforward. Corticosteroids have emerged as a standard and effective first-line therapy, with over $90 \%$ of patients achieving complete remission of proteinuria with steroid therapy essentially regardless of dose, route, and duration of therapy [1]. Despite such high remission rates, however, the management of patients with MCD can be quite complex. Patients frequently experience adverse effects of corticosteroid therapy, and a high rate of relapse further plagues the clinical course of MCD across all age spectrums, often necessitating repeat exposure to steroids and/or other, potentially toxic immunosuppressive agents [2]. Therefore, since the initial recognition that corticosteroids led to a remission of "nil disease," nephrologists have consistently sought the answer to a relatively straightforward question for MCD: What is the least amount of corticosteroids my patient needs to achieve a remission and not relapse immediately?

The answer to that question has been worked out for the pediatric MCD population over a span of more than 5 decades, and the current standard of care is a regimen of daily corticosteroids (e.g., prednisone $60 \mathrm{mg} / \mathrm{m}^{2}$ ) for 4 weeks followed by an additional 4-8 weeks of alter-

\section{KARGER}

(c) 2018 S. Karger AG, Basel

E-Mail karger@karger.com

www.karger.com/ajn nate day corticosteroids (e.g., prednisone $40 \mathrm{mg} / \mathrm{m}^{2}$ ) [3]. A number of clinical trials and a meta-analysis of these trials have found no additional benefit for children with MCD in extending corticosteroid exposure past the 2- or 3-month mark [4-7]. For adults with MCD, the same clarity does not exist. Most adults with MCD receive a significantly higher amount of corticosteroids than their pediatric counterparts, with the typical regimen involving a high initial dose (e.g., prednisone $1 \mathrm{mg} / \mathrm{kg} /$ day or $2 \mathrm{mg} / \mathrm{kg} /$ every other day) for 8 weeks followed by an additional 16 weeks of tapering, maintenance dosing of steroids. Until now, no formal, prospective investigation has asked whether adult MCD patients truly need such a long initial duration of corticosteroid therapy or whether, like children with the same disease, a much briefer treatment course might suffice.

In this issue of the American Journal of Nephrology, Ozeki et al. [8] report on a prospective, observational study to evaluate the effectiveness of a 2-month, shortterm steroid regimen in the treatment of adult, steroidsensitive MCD patients. These investigators compared the course of 35 adult patients with MCD who, after achieving complete remission quickly, were taken off steroids within 2 months (akin to pediatric MCD patients) 
to a historical cohort of 140 adult MCD patients treated with conventional, longer duration steroid regimens. They found a non-significantly higher rate of relapse in the short-term regimen group $(68.8 \%)$ compared to their historical controls (53.6\%, $p=0.1$ for comparison), as well as significantly earlier occurrences of such relapses (on average, 159 days from initial treatment versus 357 days from initial treatment, $p<0.001$ for comparison). However, taking a long-term view, they found no difference in frequent relapses (defined by these investigators as $>2$ relapses in a 6-month period) between those treated with a short- (14.3\%) vs. long-term (13.6\%) course of steroids. As a result, there was no difference between the groups in utilization of second-line immunosuppressive agents.

Overall, patients treated with the short-term corticosteroid regimen received about half the cumulative dose of patients treated with the conventional regimen, averaging 4,199.3 mg of prednisolone over 24 months of follow-up compared to $8,540.0 \mathrm{mg}$ of prednisolone over the same time period. Surprisingly, given this substantial discrepancy in corticosteroid exposure, there were no statistically significant differences in reported adverse events. Reporter bias may account for this lack of difference, however, as adverse events were prospectively surveyed among the short-term treated patients and extracted from registry records in the historical, conventionally treated cohort patients. For example, whereas most studies in the literature report a rate of infection between 5 and $15 \%$ in adults with MCD on corticosteroids [1-3], only 6 of the 140 (4.3\%) adult MCD patients in the longterm steroid group had such complications. Despite no statistically significant adverse event differences between the short- and long-term steroid groups, some clear trends have emerged including a lower incidence of infectious-related hospitalization, peptic ulcer disease, and femoral head osteonecrosis. There was also a striking (albeit non-significant) difference in the development of de novo diabetes (2.9\% among the short-term MCD patient versus $11.4 \%$ in the conventionally treated patients).

Ozeki et al. [8] conclude that a short-term steroid regimen, lifted from the pediatric nephrology practices, may represent an effective treatment option that ensures lower steroid exposure for adult MCD patients. Theirs was an interesting study that certainly added some support to this idea but was far from definitive, limited by a small number of subjects treated with the short-term regimen and a historical control group that received a longer initial duration (up to 2 years for some patients) of corticosteroids than had been used in most modern practices. These data need replication in larger cohorts of MCD drawing from patients in other parts of the world. Ideally, the question of short- versus long-term corticosteroid regimens should be asked in a randomized controlled trial. However, this small but intriguing report from 17 affiliated Japanese centers clearly raises the possibility that we are keeping adults with MCD on steroids far too long.

\section{References}

1 Hogan J, Radhakrishnan J: The treatment of minimal change disease in adults. J Am Soc Nephrol 2013;24:702-711.

2 Waldman M, Crew RJ, Valeri A, et al: Adult minimal-change disease: clinical characteristics, treatment, and outcomes. Clin J Am Soc Nephrol 2007;2:445-453.

3 Vivarelli M, Massella L, Ruggiero B, Emma F: Minimal change disease. Clin J Am Soc Nephrol 2017;12:332-345.

4 Teeninga N, Kist-van Holthe JE, van Rijswijk $\mathrm{N}$, et al: Extending prednisolone treatment does not reduce relapses in childhood nephrotic syndrome. J Am Soc Nephrol 2013;24: 149-159.

5 Sinha A, Saha A, Kumar M, et al: Extending initial prednisolone treatment in a randomized control trial from 3 to 6 months did not significantly influence the course of illness in children with steroid-sensitive nephrotic syndrome. Kidney Int 2015;87:217224.

6 Yoshikawa N, Nakanishi K, Sako M, et al: A multicenter randomized trial indicates initial prednisolone treatment for childhood nephrotic syndrome for two months is not inferior to six-month treatment. Kidney Int 2015; 87:225-232.

7 Hahn D, Hodson EM, Willis NS, Craig JC: Corticosteroid therapy for nephrotic syndrome in children. Cochrane Database Syst Rev 2015;3:CD001533.

8 Ozeki T, Katsuno T, Hayashi H, et al: Shortterm steroid regimen for adult steroid-sensitive minimal change disease. Am J Nephrol 2019;49:54-63.
Shortening the Duration of Corticosteroid Exposure in Minimal Change Disease 